\title{
Assessment of Sub-Endometrial Junction Zone by 3-Dimensional Transvaginal Ultrasound in Unexplained Recurrent Implantation Failure and Its Effect on ICSI Outcomes
}

\author{
Abd El-Naser Abd El-Gaber Ali ${ }^{*}$, Syed A. Taha1, Mohammed F. Abd El-Ghany', \\ Mustafa M. Khodry' ${ }^{1}$, Ahmed M. Abbas ${ }^{2}$ \\ ${ }^{1}$ ART Unit, Department of Obstetrics \& Gynecology, Faculty of Medicine, South Valley University, Qena, Egypt \\ ${ }^{2}$ Department of Obstetrics \& Gynecology, Faculty of Medicine, Assiut University, Assiut, Egypt \\ Email: *abdelnaser.abdelgaber@med.svu.edu.eg
}

How to cite this paper: Abd El-Gaber Ali, A.E.-N., Taha, S.A., Abd El-Ghany, M.F., Khodry, M.M. and Abbas, A.M. (2019) Assessment of Sub-Endometrial Junction Zone by 3-Dimensional Transvaginal Ultrasound in Unexplained Recurrent Implantation Failure and Its Effect on ICSI Outcomes. Open Journal of Obstetrics and Gynecology, 9, 54-61.

https://doi.org/10.4236/ojog.2019.91006

Received: December 19, 2018

Accepted: January 11, 2019

Published: January 14, 2019

Copyright ( 2019 by author(s) and Scientific Research Publishing Inc. This work is licensed under the Creative Commons Attribution International License (CC BY 4.0).

http://creativecommons.org/licenses/by/4.0/

\begin{abstract}
BACKGROUND: Sub-endometrial junction zone (JZ) plays an important role in most of reproductive functions. OBJECTIVE: To find out the effect of the sub-endometrial JZ thickness assessment by 3-D trans-vaginal ultrasound (TVUS) on intracytoplasmic sperm injection (ICSI) outcomes in patients with unexplained recurrent implantation failure (RIF). SETTING: ART Unit of Obstetrics and Gynecology Department, Faculty of Medicine, South Valley University, Qena, Egypt. DURATION: From April 2016 to October 2018. STUDY DESIGN: A prospective observational study. METHDS: Fifty couples with history of unexplained RIF in previous ICSI cycles and prepared for another ICSI cycle (group I) and fifty couples with unexplained infertility prepared for ICSI for the first time (group II) had been included in this study. At time of ovum pick up, 3-DTVUS was done for all cases in both groups for assessment of sub endometrial junction zone thickness and correlated with ICSI outcome. RESULTS: There were statistically significant differences between group I and group II in JZ thickness in the 3 uterine regions (fundus, anterior and posterior walls) with $\mathrm{p}<0.001$, but there were mildly statistically significant differences between both groups in chemical and clinical pregnancy rates with $p<0.01$. CONCLUSIONS: The thickness of JZ in patients with history of unexplained RIF was higher than those with unexplained infertility scheduled for ICSI. The JZ thickness was inversely correlated with increased embryo implantation rates in ICSI procedures, the thinner the JZ thickness was associated with higher pregnancy rates (both chemical and clinical pregnancy rates).
\end{abstract}




\section{Keywords}

Three Dimensional Trans-Vaginal Ultrasound, Junction Zone, ICSI, Recurrent Implantation Failure

\section{Introduction}

Successful embryo implantation depends on interaction between the endometrium and the blastocyst. The thickness of endometrium and the good uterine blood supply are considered in pregnancy enhancement. Also, increased endometrial and subendometrial blood flow had been found to be higher in women with live births after Assisted Reproductive Technology (ART) than in those who had suffered from miscarriage.

Anyway, conflicting results were reported as regard to its importance in the prediction of successful pregnancy in ART procedure [1]. Uterine junction zone (JZ) can play an important integral part in most reproductive functions. The junction zone also can be called archimyometrium or stratum subvasculare, which is the inner third of myometrium and is located at the interface between endometrium and the myometrium. It can be considered as a hormone-dependent structure, under cyclic change affected by estrogen and progesterone throughout the menstrual cycle as comparable with the changes seen in the endometrium.

The major function of the junction zone throughout the women reproductive life is to direct sperm transport toward the ipsilateral tube to dominant follicle, which can be provided by uterine peristalsis of junction zone [2]. Furthermore, it had been proven that an optimal thickness of the junction zone was mandatory in the successful embryo implantation. A thickened uterine junction zone on magnetic resonance imaging was a failure predictive agent for embryo implantation in the intracytoplasmic (ICSI) or in vitro fertilization (IVF) trials [3]. Three dimensional trans-vaginal ultrasound (3-D TV US) can produce a volume of data including any anatomical plane of the uterus. This can allow reconstructed coronal sections through the uterus which is commonly done with 3-D TV US, which could not be seen with 2D TVU/S [4].

\section{Aim of Work}

The aim of this work was to find out the role of the sub-endometrial junction zone thickness assessment by 3D TVUS in ICSI outcome in patients with unexplained recurrent implantation failure in previous ICSI cycles.

\section{Patients and Methods}

This study was performed at the ART Unit of Obstetrics and Gynecology Department, Qena, South Valley University during the period (April 2016 to October 2018). A pilot study included Fifty couples with history of unexplained RIF 
in previous ICSI procedures and prepared for another ICSI cycle (group I) and fifty couples with unexplained infertility prepared for ICSI procedure for first time (group II) had been enrolled in this study. A good clear counseling of all couples who had been included in this study was done beside a written consent was obtained from them that agreed with the medical ethics committee of faculty of medicine, south valley university.

Inclusion criteria: (a) recurrent implantation failure in previous ICSI procedures for group I (b) unexplained infertility for group II

Exclusion criteria: 1) cases with history of miscarriage 2) male factor of infertility 3) any obvious cause of female infertility as ovarian or uterine factors 4) uterine adenomyosis 5) uterine fibroids 6) pelvic endometriosis 7) irregular menstrual cycle 8) any medical disorder or endocrine gland disorders as hypo or hyperthyroidism.

\subsection{Methodology}

All cases underwent routine work up of infertility and laboratory investigations including ovarian reserve tests (basal FSH, basal E2, basal AFC and AMH), uterine cavity assessment with 3DTVUS or office hysteroscopy and husband semen profile. All cases received combined contraceptive pills for 2 to 3 weeks and at the second day of menstruation, gonadotrophins stimulation was started, (recombinant FSH; Gonal-F pen subcutaneously; Serono, Aubonne, Switzerland) and hMG (Menogon; Ferring 75 IU intramuscularly) at 300 - 450 IU/day.

Patient monitoring for follicular growth with trans-vaginal ultrasound started at day 5 of stimulation and repeated each other day with estradiol estimation. When 2 or more follicles in both ovaries achieved size of $14 \mathrm{~mm}$ or more in mean diameter, antagonist pituitary suppression protocol was started (Cetrotide $0.25 \mathrm{mg}$ Merck Serono), which was daily injected subcutaneously till triggering time (10,000 IU of Human chorionic gonadotrophins was intramuscularly injected), Cancellation of the cycle was done if 2 follicles or less only could achieve normal maturation.

Ovum pick up was done exactly 36 hours post trigger injection. At time of ovum pick up 3D TVUS was done for all cases in both groups for sub endometrial junction zone thickness assessment.

Technique: in lithotomy position with empty bladder, all patients had a conventional TVUS examination using a Sonoace X8, Medison ultrasound system (Seoul, South Korea) equipped with a $6-12 \mathrm{MHz}$ 3D transvaginal probe. The 3 $\mathrm{D}$ volume box was applied covering the entire whole uterus and then a $3 \mathrm{D}$ volume had been generated by automatic sweep of the mechanical probe. The volume had been stored digitally and analyzed off-line. The junction zone appeared as a hypoechoic zone around the hyperechoic endometrium on the coronal sight (Figure 1). With VCI modality with 2 to $4 \mathrm{~mm}$ slices the sub-endometrial junction zone was seen in all the multilane view planes and so its thickness could be measured as the distance between the lower edge of endometrium to the inner layer of the outer myometrium [5]. 


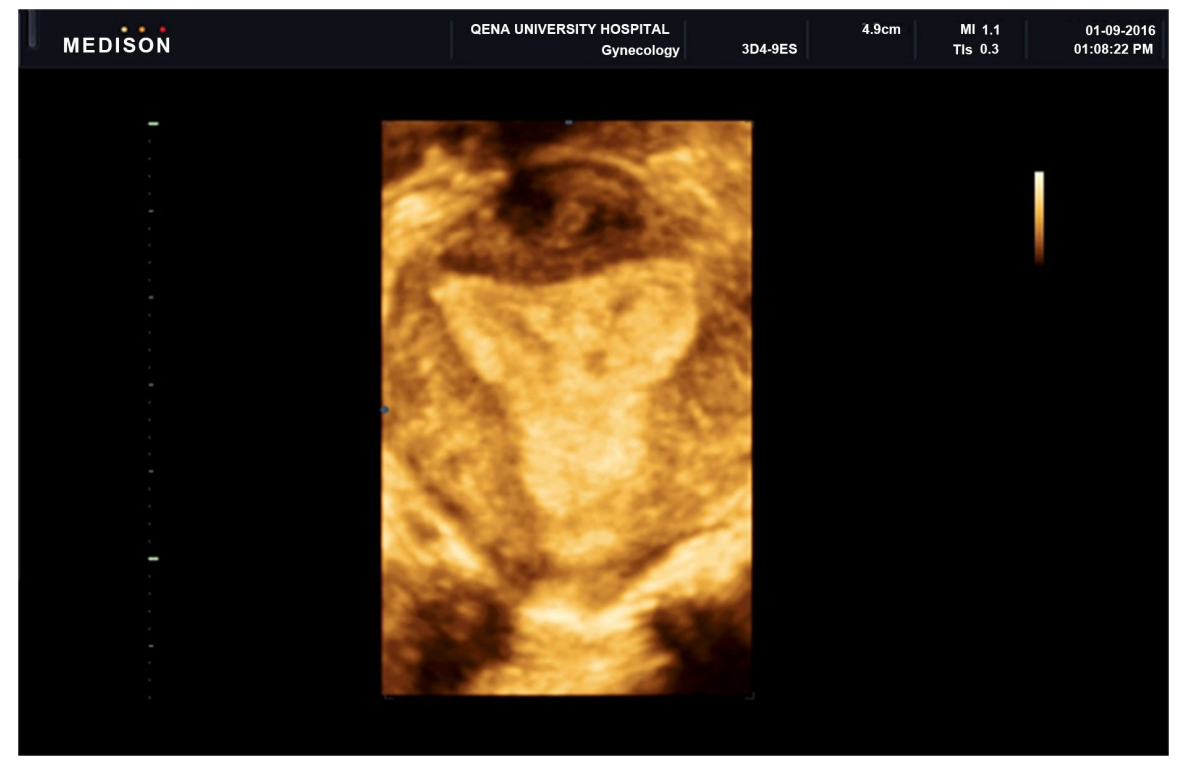

Figure 1. 3-D TV US showing sub endometrial junction zone in coronal view of the uterus.

Sonographic images which had been obtained on 3-D US multiplane view and the volume contrast imaging had been expressed as: Junction Zone maximum (JZ max) and Junction Zone minimum (JZ min) diameters where the largest and smallest junction zone thickness were measured on the coronal plane or on the longitudinal plane at 3 regions of the uterus (fundus, right and left sides) [6]. The assessment of all patients was done by one sonographer with good experience of 3-D ultrasound assessment.

For all cases, 1 - 3 (day 3 to day 5) embryos of good quality (grades A and B) only were allowed to be transferred. Luteal phase supports with exogenous progesterone was started at after oocytes retrieval and continue for the next 2 weeks which pregnancy tests were done and positive pregnancy cases continued luteal support till the end of first trimester of pregnancy. Finally data was collected, recorded and analyzed collectively.

\subsection{Statistical Analysis}

Analysis and statistical presentation of this study were conducted, using the mean, standard error, unpaired student t-test, and chi-square tests using the Statistical Program for Social Sciences (SPSS Inc., Version 21.0, Chicago, IL, USA). A two sided $\mathrm{p}<0.05$ was considered to be statistically significant.

\section{Results}

In group I; 6 cases (12\%) were cancelled from the ICSI cycle; 3 cases (6\%) due to failure of follicular maturation, one case (2\%) had no oocytes retrieved and 2 cases (4\%) failed to be fertilized. As regard to group II; 2 cases (4\%) were cancelled from the cycle; one case $(2 \%)$ due to failure of follicular maturation and the other due to failure of fertilization. 
There was no statistically significant difference between group I and group II as regard to women age, body mass index, basal FSH, basal E2, AMH and basal AFC with $\mathrm{p}>0.05$ (Table 1 ). There was no statistically significant difference between group I and group II as regard to the duration and doses of gonadotrophins stimulation, number of retrieved oocytes, endometrial thickness or embryos quality with $\mathrm{p}>0.05$ (Table 2 ).

There were a statistically significant differences between group I and group II in JZ thickness in the 3 uterine regions (fundus, anterior and posterior walls) with $\mathrm{p}<0.001$ (Table 3 ). Additionally, the mean JZ average in group II was significanctly lower than group I $(0.31 \pm 0.09$ versus $0.38 \pm 0.11 \mathrm{~mm}, \mathrm{p}<0.001)$.

As regard to pregnancy rates, there were mildly statistically significant differences between both groups in chemical and clinical pregnancy rates $(\mathrm{p}<0.01)$ (Figure 2).

\section{Discussion}

The sub endometrial junction zone could be identical to the endometrium as regard to its embryological origin, where the Müllerian duct (paramesonephric duct) is in contrast to the rest of the myometrium (mesenchymal) [7]. The physiological role of JZ thickness in implantation rate still unclear however JZ might be involved in the subtle contractile activities of myometrium that is reflected by the endometrial wave-like movements that detected by TVUS [8]. Besides the higher vascularity of the JZ which was proved by anti-CD31 stains could be involved in the explanation of different JZ thickness associated with different implantation success rates [9].

Sometimes many cases have RIF in ICSI cycles with no cause could be detected, many previous reports took about the subendometrial junction zone and its role in successful embryos implantation rates in ICSI procedures as what was reported by Maged et al. [10] which assessed the JZ thickness with 3-D TVUS for patients during ICSI procedure twice (at time of pituitary down regulation and at time of ovum pick up) and concluded that the pregnant cases had a thinner JZ $(0.29 \pm 0.08)$ than non-pregnant cases $(0.37 \pm 0.09)$ with a highly statistically significance at time of ovum pick up $(\mathrm{p}=0.002)$ but had no significant difference at time of pituitary down regulation with $\mathrm{p}>0.05$.

Table 1. The baseline characteristics of the study groups.

\begin{tabular}{cccc}
\hline & Group I $(\mathbf{n}=44)$ & Group II $(\mathbf{n}=48)$ & p-value \\
\hline Age in years $($ mean $\pm \mathrm{SD})$ & $27.8 \pm 3.4$ & $28.7 \pm 5.2$ & $>0.05$ \\
BMI in $\mathrm{Kg} / \mathrm{m}^{2}($ mean $\pm \mathrm{SD})$ & $23.5 \pm 3.1$ & $24.1 \pm 3.4$ & $>0.05$ \\
Basal FSH $(\mathrm{mIU} / \mathrm{mL})($ mean $\pm \mathrm{SD})$ & $6.7 \pm 3.7$ & $6.6 \pm 3.4$ & $>0.05$ \\
AMH ng/ml $(\mathrm{mean} \pm \mathrm{SD})$ & $3.9 \pm 1.3$ & $4.1 \pm 1.6$ & $>0.05$ \\
Basal Estradiol $(\mathrm{pg} / \mathrm{mL})($ mean $\pm \mathrm{SD})$ & $33.2 \pm 6.3$ & $34.4 \pm 8.1$ & $>0.05$ \\
Basal AFC $($ mean $\pm \mathrm{SD})$ & $7.7 \pm 2.3$ & $8.1 \pm 2.5$ & $>0.05$ \\
\hline
\end{tabular}

NS $=$ non-significant $(\mathrm{p}$ value $>0.05)$. 
Table 2. Comparison between study groups as regarding ICSI cycle characteristics.

\begin{tabular}{cccc}
\hline & $\begin{array}{c}\text { Group I } \\
(\mathbf{n}=\mathbf{4 4})\end{array}$ & $\begin{array}{c}\text { Group II } \\
(\mathbf{n}=\mathbf{4 8})\end{array}$ & p-value \\
\hline Duration of stimulation (days) mean \pm SD & $14.3 \pm 2.3$ & $13.7 \pm 2.1$ & $>0.05$ \\
Doses of gonadotrophin (IU) mean \pm SD & $4784.5 \pm 1872.1$ & $4872.3 \pm 1963.5$ & $>0.05$ \\
Number of retrieved oocytes mean \pm SD & $10.5 \pm 1.7$ & $11.1 \pm 1.8$ & $>0.05$ \\
Endometrial thickness mean \pm SD & $9.7 \pm 2.3$ & $9.1 \pm 2.0$ & $>0.05$ \\
Embryo quality No (\%) & & & \\
A \& B & $69.7 \%$ & $70.2 \%$ & $>0.05$ \\
C \& D & $30.3 \%$ & $29.8 \%$ & \\
\hline
\end{tabular}

NS $=$ non-significant $(\mathrm{p}$ value $>0.05)$.

Table 3. Comparison between Group I and Group II as regard to JZ thickness.

\begin{tabular}{cccc}
\hline & Group I $(\mathrm{n}=44)$ & Group II $(\mathrm{n}=48)$ & p-value \\
\hline Junction zone thickness in mm (mean $\pm \mathrm{SD})$ & & & \\
\hline JZ of fundus & $0.41 \pm 0.16$ & $0.32 \pm 0.12$ & $<0.001^{*}$ \\
JZ of anterior wall & $0.38 \pm 0.10$ & $0.30 \pm 0.09$ & $<0.001^{*}$ \\
JZ of posterior wall & $0.35 \pm 0.08$ & $0.33 \pm 0.07$ & $<0.001^{*}$ \\
JZ average & $0.38 \pm 0.11$ & $0.31 \pm 0.09$ & $<0.001^{*}$ \\
\hline
\end{tabular}

${ }^{*}$ Highly statistically significant difference.

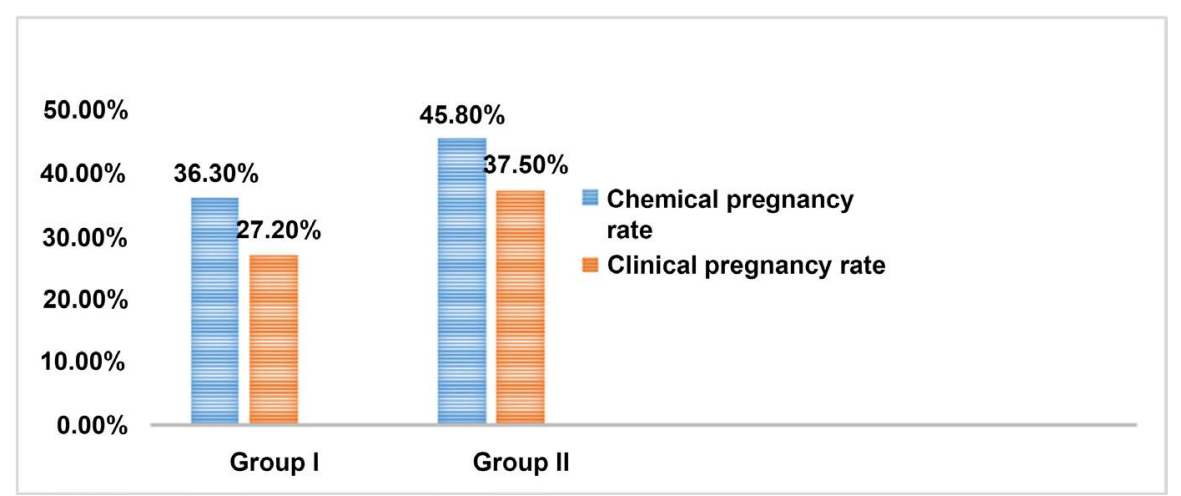

Figure 2. Comparison between Group I and Group II as regard to chemical and clinical pregnancy rates, $\mathrm{p}$ value $<0.01$.

In our study we had tried to check the role of JZ thickness measured by $3 \mathrm{D}$ TVUS at time of ovum pick up on ICSI outcome for patients with past history of unexplained RIF (group I) and compared to JZ for patient with unexplained infertility (group II) prepared for ICSI for first time. in our study we found no significant differences between both groups regard to the duration and doses of gonadotrophins stimulation, number of number of retrieved oocytes, endometrial thickness at time of ovum pick up or in the embryos qualities, but as regard to junction zone thickness we had found that the average JZ thickness was significantly higher in patients with recurrent implantation failure (group I) (0.38 \pm 
$0.11)$ than in group II $(0.31 \pm 0.09)(\mathrm{P}<0.001)$. Also, pregnancy rates (chemically and clinically) had moderate significant differences between both groups (45.8\% and $37.5 \%$ in group II versus $36.3 \%$ and $27.2 \%$ in group I) (p $<0.01$ ). These results agreed with what had been reported by Maged et al. [10]. The results of our study could explain the important role of sub endometrial junction zone in the success of embryo implantation in ICSI cycles and patients with unexplained RIF in previous ICSI cycle might due to their thicker JZ thickness which had a negative impact on implantation rates.

Maubon et al. [3] discussed the uterine JZ effect on IVF results for infertile women and had used pelvic MRI scanning in the JZ thickness assessment and had showed that a thickened uterine JZ was a failure predictive factor for the embryo implantation in IVF trials. In Maubon et al. study [3], the increase in JZ thickness was significantly correlated with the implantation failure in IVF (rate of implantation failure was $95.8 \%$ for cases with average JZ more than $7 \mathrm{~mm}$ and maximal JZ more than $10 \mathrm{~mm}$, versus $37.5 \%$ in the other groups with $\mathrm{p}<0.0001$, apart from the causes of infertility or women' age.

Actually the previous study based on MRI assessment of JZ and our study based on 3D US and there are big discrepancies between MRI and ultrasound in the JZ assessment so it is not fair to compare our results with Maubon et al. results and that opinion agreed with what had been reported by Mitchell et al. [11]. As regard to the cost effectiveness of this procedure, it is considered less inexpensive procedure (in comparison to MRI) could be beneficial in the success of ICSI trials.

Ultrasound machines with high resolution could provide new insights into the process of implantation and to assess functional changes in JZ. Any defect in the process of implantation can lead to early or late pregnancy loss, obstetrical disorders that associated with improper placentation as preeclampsia, placental abruption or fetal growth restriction; it could be possible for early prediction of complications at the implantation stage and so design of interventions which are needed for its prevention [12].

The main study limitation was the small sample size as we considered it as a pilot study in our center. Future trials are running nowadays with adequate power based on the results of the current study to confirm our conclusions.

\section{Conclusions}

1) The thickness of JZ in patients with history of unexplained RIF was higher than those with unexplained infertility.

2) The JZ thickness was inversely correlated with increased embryo implantation rates in ICSI procedures, the thinner the JZ thickness was associated with higher pregnancy rates (both chemical and clinical pregnancy rates).

\section{Conflicts of Interest}

The authors declare that they have no conflict of interest. 


\section{References}

[1] Yaman, C. and Mayer, R. (2012) Three-Dimensional Ultrasound as a Predictor of Pregnancy in Patients Undergoing ART. Journal Of The Turkish-German Gynecological Association, 13, 28-34. https://doi.org/10.5152/jtgga.2012.15

[2] Meylaerts, L.J., Wijnen, L., Ombelet, W., Bazot, M. and Vandersteen, M. (2017) Uterine Junctional Zone Thickness in Infertile Women Evaluated by MRI. Journal of Magnetic Resonance Imaging, 45, 926-936. https://doi.org/10.1002/jmri.25422

[3] Maubon, A., Faury, A., Kapella, M., Pouquet, M. and Piver, P. (2010) Uterine Junctional Zone at Magnetic Resonance Imaging: A Predictor of in Vitro Fertilization Implantation Failure. Journal of Obstetrics and Gynaecology Research, 36, 611-618. https://doi.org/10.1111/j.1447-0756.2010.01189.x

[4] Kerr, N.K., Dunham, R., Wolstenhulme, S. and Wilson, J. (2014) Comparison of Two- and Three-Dimensional Transvaginal Ultrasound in the Visualisation of Intrauterine Devices. Ultrasound, 22, 141-147.

https://doi.org/10.1177/1742271X14532082

[5] Exacoustos, C., Brienza, L., Di Giovanni, A., Szabolcs, B., Romanini, M.E., Zupi, E., et al. (2011) Adenomyosis: Three-Dimensional Sonographic Findings of the Junctional Zone and Correlation with Histology. Ultrasound in Obstetrics \& Gynecology, 37, 471-479. https://doi.org/10.1002/uog.8900

[6] Exacoustos, C., Luciano, D., Corbett, B., DeFelice, G., DiFeliciantonio, M., Luciano, A., et al. (2013) The Uterine Junctional Zone: A 3-Dimensional Ultrasound Study of Patients with Endometriosis. American Journal of Obstetrics and Gynecology, 209, e1-e7. https://doi.org/10.1016/j.ajog.2013.06.006

[7] Noe, M., Kunz, G., Herbertz, M., Mall, G. and Leyendecker, G. (1999) The Cyclic Pattern of the Immunocytochemical Expression of Estrogen and Progesterone Receptors in Human Myometrial and Endometrial Layers: Charcterization of the Endometrial-Subendometrial Unit. Human Reproduction, 14, 190-197.

https://doi.org/10.1093/humrep/14.1.190

[8] Birnholz, J.C. (1984) Ultrasonic Visualisation of Endometrial Movements. Fertility and Sterility, 41, 157-158. https://doi.org/10.1016/S0015-0282(16)47560-6

[9] Tetlow, R.L., Richmond, I., Manton, D.J., Greenman, J., Turnbull, L.W. and Killick, S.R. (1999) Histological Analysis of the Uterine Junctional Zone as Seen by Transvaginal Ultrasound. Ultrasound in Obstetrics and Gynecology, 14, 188-193. https://doi.org/10.1046/j.1469-0705.1999.14030188.x

[10] Maged, A.M., Ramzy, A.M., Ghar, M.A., El Shenoufy, H., Allah, S.H., Wahba, A.H., et al. (2017) 3D Ultrasound Assessment of Endometrial Junctional Zone Anatomy as a Predictor of the Outcome of ICSI Cycles. European Journal of Obstetrics \& Gynecology and Reproductive Biology, 212, 160-165. https://doi.org/10.1016/j.ejogrb.2017.03.035

[11] Mitchell, D.G., Schonholz, L., Hilpert, P.L., Pennell, R.G., Blum, L. and Rifkin, M.D. (1990) Zones of the Uterus: Discrepancy between US and MR Images. Radiology, 174, 827-831. https://doi.org/10.1148/radiology.174.3.2406787

[12] Abdallah, Y., Naji, O., Saso, S., Pexsters, A., Stalder, C., Sur, S., et al. (2012) Ultrasound Assessment of the Peri-Implantation Uterus: A Review. Ultrasound in $\mathrm{Ob}$ stetrics \& Gynecology, 39, 612-619. https://doi.org/10.1002/uog.10098 